\title{
Optical band gap and magnetic properties of unstrained $\mathrm{EuTiO}_{3}$ films
}

J. H. Lee, X. Ke, N. J. Podraza, L. Fitting Kourkoutis, T. Heeg, M. Roeckerath, J. W. Freeland, C. J. Fennie, J. Schubert, D. A. Muller, P. Schiffer, and D. G. Schlom

Citation: Appl. Phys. Lett. 94, 212509 (2009);

View online: https://doi.org/10.1063/1.3133351

View Table of Contents: http://aip.scitation.org/toc/apl/94/21

Published by the American Institute of Physics

\section{Articles you may be interested in}

High-quality antiferromagnetic $\mathrm{EuTiO}_{3}$ epitaxial thin films on $\mathrm{SrTiO}_{3}$ prepared by pulsed laser deposition and postannealing

Applied Physics Letters 94, 062512 (2009); 10.1063/1.3072598

Magnetic Structure of $\mathrm{EuTiO}_{3}$

Journal of Applied Physics 37, 981 (2004); 10.1063/1.1708549

Conduction mechanisms of epitaxial $\mathrm{EuTiO}_{3}$ thin films

Applied Physics Letters 101, 102901 (2012); 10.1063/1.4750073

Evidence of $\mathrm{Eu}^{2+} 4 \mathrm{f}$ electrons in the valence band spectra of $\mathrm{EuTiO}_{3}$ and $\mathrm{EuZrO}_{3}$

Journal of Applied Physics 112, 083719 (2012); 10.1063/1.4761933

High pressure antiferrodistortive phase transition in mixed crystals of $\mathrm{EuTiO}_{3}$ and $\mathrm{SrTiO}_{3}$

AIP Advances 6, 065019 (2016); 10.1063/1.4954819

Magnetocaloric properties of $\mathrm{Eu}_{1-x} \mathrm{La}_{x} \mathrm{TiO}_{3}(0.01 \leq \mathrm{x} \leq 0.2)$ for cryogenic magnetic cooling Journal of Applied Physics 119, 243901 (2016); 10.1063/1.4954020

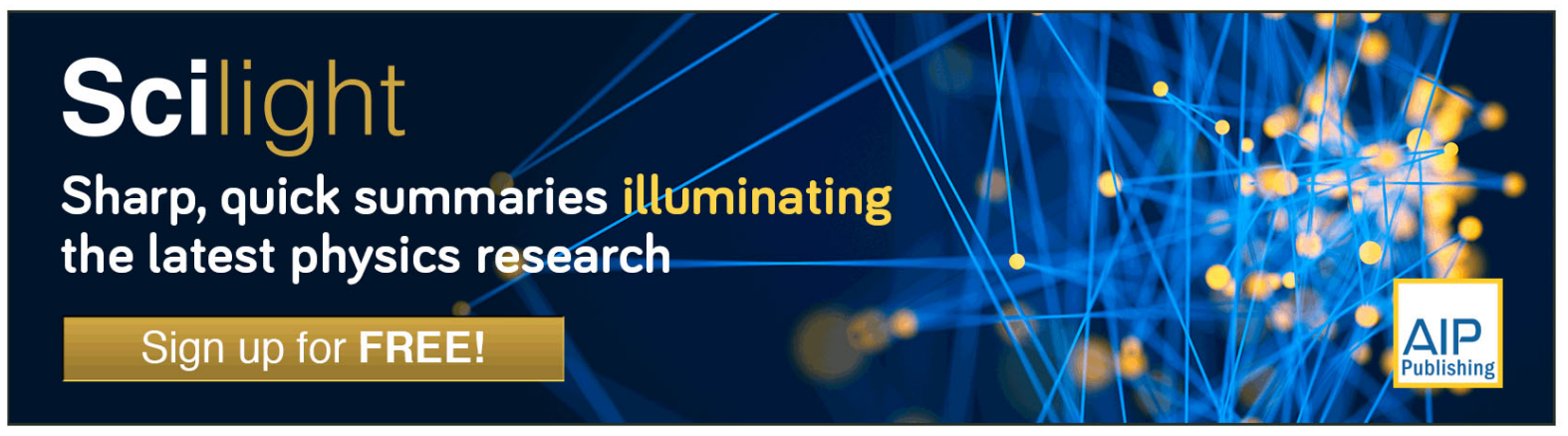




\title{
Optical band gap and magnetic properties of unstrained $\mathrm{EuTiO}_{3}$ films
}

\author{
J. H. Lee, ${ }^{1,2}$ X. Ke ${ }^{3}$ N. J. Podraza, ${ }^{4}$ L. Fitting Kourkoutis,${ }^{5}$ T. Heeg, ${ }^{1}$ M. Roeckerath, ${ }^{6}$ \\ J. W. Freeland, ${ }^{7}$ C. J. Fennie, ${ }^{5}$ J. Schubert, ${ }^{6}$ D. A. Muller, ${ }^{5}$ P. Schiffer, ${ }^{3}$ and \\ D. G. Schlom ${ }^{1, a)}$ \\ ${ }^{1}$ Department of Materials Science and Engineering, Cornell University, Ithaca, New York 14853, USA \\ ${ }^{2}$ Department of Materials Science and Engineering, Pennsylvania State University, University Park, \\ Pennsylvania 16802, USA \\ ${ }^{3}$ Department of Physics, Pennsylvania State University, University Park, Pennsylvania 16802, USA \\ ${ }^{4}$ Department of Electrical Engineering, Pennsylvania State University, University Park, \\ Pennsylvania 16802, USA \\ ${ }^{5}$ School of Applied and Engineering Physics, Cornell University, Ithaca, New York 14853, USA \\ ${ }^{6}$ Institute of Bio- and Nano-Systems, IBN-1, Jülich GmbH and Jülich-Aachen Research Alliance, JARA-FIT, \\ Jülich 52425, Germany \\ ${ }^{7}$ Advanced Photon Source, Argonne National Laboratory, Argonne, Illinois 60439, USA
}

(Received 23 February 2009; accepted 20 April 2009; published online 28 May 2009)

\begin{abstract}
Phase-pure, stoichiometric, unstrained, epitaxial (001)-oriented $\mathrm{EuTiO}_{3}$ thin films have been grown on (001) $\mathrm{SrTiO}_{3}$ substrates by reactive molecular-beam epitaxy. Magnetization measurements show antiferromagnetic behavior with $T_{N}=5.5 \mathrm{~K}$, similar to bulk $\mathrm{EuTiO}_{3}$. Spectroscopic ellipsometry measurements reveal that $\mathrm{EuTiO}_{3}$ films have a direct optical band gap of $0.93 \pm 0.07 \mathrm{eV}$. () 2009 American Institute of Physics. [DOI: 10.1063/1.3133351]
\end{abstract}

$\mathrm{EuTiO}_{3}$ has many similarities to $\mathrm{SrTiO}_{3}$ including being a quantum paraelectric ${ }^{1,2}$ and a cubic structure (space group $P m \overline{3} m$ ) with a lattice parameter of $3.905 \AA$ at room temperature. $^{2-5}$ In contrast to the diamagnetic nature of $\mathrm{SrTiO}_{3}$, bulk $\mathrm{EuTiO}_{3}$ exhibits antiferromagnetic order at $5 \mathrm{~K}$ due to the localized $4 f$ moments on the $\mathrm{Eu}^{2+}$ site. ${ }^{6,7}$ While $\mathrm{SrTiO}_{3}$ is on the brink of being ferroelectric, $\mathrm{EuTiO}_{3}$ is on the brink of being simultaneously ferroelectric and ferromagnetic. $^{8,9}$

With its nearly identical lattice constant, $\mathrm{SrTiO}_{3}$ has been shown to be an excellent substrate for the growth of epitaxial $\mathrm{EuTiO}_{3}$ films. ${ }^{10}$ Significantly different structural and magnetic properties, however, have been reported for $\mathrm{EuTiO}_{3}$ films. As-grown $\mathrm{EuTiO}_{3-\delta}$ films by pulsed-laser deposition (PLD) on (001) $\mathrm{SrTiO}_{3}$ substrates exhibit expanded out-of-plane spacings $(0.4 \%-2 \%$ longer than bulk $\left.\mathrm{EuTiO}_{3}\right)^{10-13}$ and are ferromagnetic with a Curie temperature of about $5 \mathrm{~K}^{11,12}$ Biaxial strain has been predicted to cause significant changes to the properties of $\mathrm{EuTiO}_{3}$, including the emergence of a multiferroic ground state where $\mathrm{EuTiO}_{3}$ is simultaneously ferromagnetic and ferroelectric at sufficiently high values of strain ( $\geq 1.2 \%$ biaxial compression). ${ }^{8}$ As the lattice constant of $\mathrm{EuTiO}_{3}$ is identical to that of $\mathrm{SrTiO}_{3}$ within $<0.1 \%$, the epitaxial growth of fully oxygenated $\mathrm{EuTiO}_{3}$ films is not expected to yield ferromagnetic films or films with extended lattice constants. The negligible $(<0.5 \%)$ variation in the cubic lattice constant of oxygen deficient $\mathrm{EuTiO}_{3-\delta}$ over its wide single phase field, ${ }^{14,15}$ up to the $\mathrm{EuTiO}_{2.5}$ limit $^{14}$ of the perovskite $\mathrm{EuTiO}_{3-\delta}$ structure, is insufficient to explain the $2 \%$ elongation observed in epitaxial $\mathrm{EuTiO}_{3-\delta}$ films grown on (001) $\mathrm{SrTiO}_{3}$ by PLD. ${ }^{11-13}$

One possibility is that the ferromagnetism observed in epitaxial $\mathrm{EuTiO}_{3}$ films on $\mathrm{SrTiO}_{3}$ arises from extrinsic effects. Extrinsic effects are known to occur in thin films, particularly for deposition technologies involving energetic spe-

${ }^{a)}$ Electronic mail: schlom@cornell.edu. cies, which can induce defects. For example, some homoepitaxial $\mathrm{SrTiO}_{3}$ films grown by PLD have been reported to be ferroelectric ${ }^{16}$ in striking contrast to the intrinsic properties of $\mathrm{SrTiO}_{3}$, which is not ferroelectric at any temperature. ${ }^{1}$ Homoepitaxial $\mathrm{SrTiO}_{3}$ films grown by PLD are also known to exhibit lattice spacings that deviate significantly from the $\mathrm{SrTiO}_{3}$ substrates they are grown on. ${ }^{17,18}$ Such issues lead to the question of what the intrinsic properties of unstrained $\mathrm{EuTiO}_{3}$ films really are.

To clarify the different ferromagnetic orderings reported for bulk and thin film $\mathrm{EuTiO}_{3}$, the growth of high crystalline quality epitaxial thin films is desired. Reactive molecularbeam epitaxy (MBE) has been successful in the preparation of high quality thin films of related oxides including $\mathrm{SrTiO}_{3}$, $\mathrm{BaTiO}_{3}$, and $\mathrm{EuO}$ by optimizing the independent growth parameters including oxygen partial pressure. ${ }^{19}$ In this letter, we report the epitaxial growth of unstrained $\mathrm{EuTiO}_{3}$ thin films on (001) $\mathrm{SrTiO}_{3}$ by reactive $\mathrm{MBE}$ and discuss the resulting structure composition, magnetic ordering, band gap, and complex dielectric function.

$\mathrm{EuTiO}_{3}$ films were grown on buffered-HF treated $\mathrm{SrTiO}_{3}$ (001) substrates $^{20}$ in a reactive MBE system. Molecular beams of europium and titanium were generated using a conventional effusion cell and a Ti-Ball ${ }^{\mathrm{TM}}$ titanium sublimation pump, ${ }^{21}$ respectively. Europium and titanium were codeposited onto the substrate under an oxygen background partial pressure of $3 \times 10^{-8}$ Torr at a substrate temperature of $650{ }^{\circ} \mathrm{C}$. A thin layer $(\sim 10 \mathrm{~nm}$ thick) of amorphous silicon was deposited on some of the $\mathrm{EuTiO}_{3}$ films to prevent oxidation of $\mathrm{Eu}^{2+}$ into $\mathrm{Eu}^{3+}$ upon exposure to air.

The structural perfection of a silicon-capped, $22 \mathrm{~nm}$ thick $\mathrm{EuTiO}_{3}$ film grown on (001) $\mathrm{SrTiO}_{3}$ was characterized by $x$-ray diffraction (XRD). As Fig. 1(a) shows, an out-ofplane $\theta-2 \theta$ scan reveals phase-pure, epitaxial (001)-oriented $\mathrm{EuTiO}_{3}$. The $00 l$ reflections from the film are buried by the high intensity of the substrate $00 l$ reflections because the lattice constant of $\mathrm{EuTiO}_{3}$ is equal to that of $\mathrm{SrTiO}_{3}(a$ $=3.905 \AA) .{ }^{3,4}$ An $\omega$ rocking curve around the 002 peak (not 

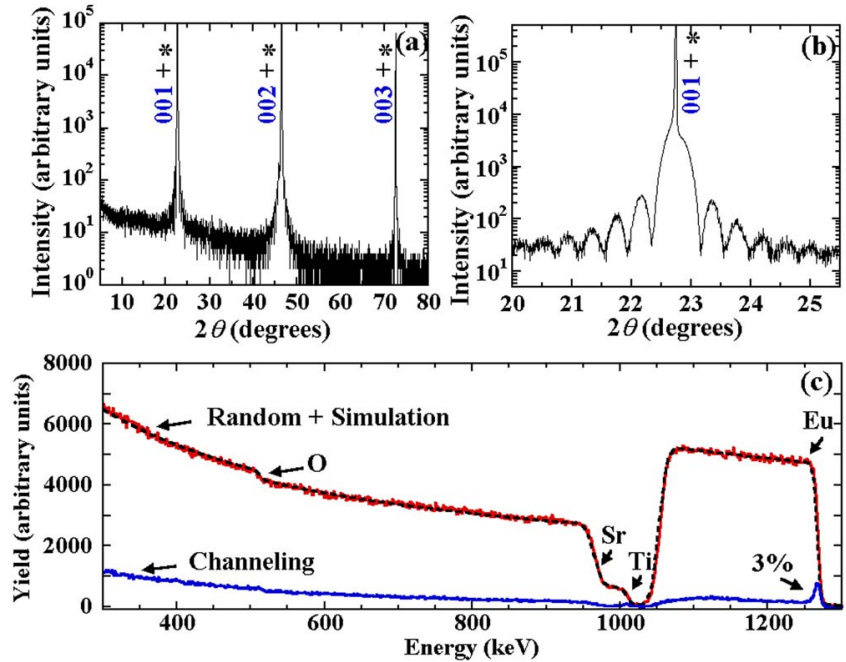

FIG. 1. (Color online) (a) $\theta-2 \theta$ XRD scans of a silicon-capped, $22 \mathrm{~nm}$ thick (001)-oriented $\mathrm{EuTiO}_{3}$ on (001) $\mathrm{SrTiO}_{3}$. Substrate peaks are marked with an asterisk (*). (b) A close-up of the 001 peak, showing thickness fringes. (c) RBS random and channeling spectra of an uncapped, $200 \mathrm{~nm}$ thick $\mathrm{EuTiO}_{3}$ film on (001) $\mathrm{SrTiO}_{3}$ with the simulated curve (dashed line). The simulated curve gives a thickness of $200 \mathrm{~nm}$ and a Eu:Ti ratio of 1:1.

shown) also reveals a single reflection from the $\mathrm{SrTiO}_{3}$ substrate. Clear thickness fringes corresponding to a film thickness of $22 \pm 1 \mathrm{~nm}$ are observed from a high-resolution $\theta-2 \theta$ scan of the $001 \mathrm{EuTiO}_{3}+001 \mathrm{SrTiO}_{3}$ peak in Fig. 1(b). These Kiessig fringes indicate the presence of a lattice-matched unstrained $\mathrm{EuTiO}_{3}$ film with a smooth film surface. Figure 1(c) shows the RBS and channeling spectra of an uncapped, 200 nm thick $\mathrm{EuTiO}_{3}$ film utilizing $\mathrm{He}^{+}$ions with an energy of 1.4 MeV. The overlaid results of a RUMP ${ }^{22}$ simulation with the RBS spectra reveal a Eu:Ti ratio of 1:1, indicating that the film composition is stoichiometric within experimental error $( \pm 5 \%)$. The low channeling minimum $\chi_{\min }=3 \%$ confirms the good crystallinity of the film.

Figures 2(a) and 2(b) show high-angle annular dark field (HAADF) images from a scanning transmission electron microscope (STEM) of the same film analyzed in Fig. 1(a). The entire epitaxial heterostructure is observed, and the interface between the film and the substrate is atomically sharp. The valence state of europium in the same film was analyzed by $\mathrm{x}$-ray absorption spectroscopy (XAS) at beamline 4-ID-C of the Advanced Photon Source. The XAS data in Fig. 2(c) show a behavior consistent with europium in the $2+$ valence state. Comparison with calculations allows us to determine that $\mathrm{Eu}^{3+}$ is below the detectable level, and from this we can confirm that the film contains $>90 \% \mathrm{Eu}^{2+} .{ }^{23}$

Contrary to prior reports of ferromagnetic behavior in PLD-grown $\mathrm{EuTiO}_{3-\delta}$ films, ${ }^{11,12}$ the MBE-grown $\mathrm{EuTiO}_{3-\delta}$ films exhibit a clear magnetic transition with a small magnetic response consistent with antiferromagnetic order. Figure 3 shows the temperature dependence of the magnetization (after subtracting the diamagnetic contribution of the $\mathrm{SrTiO}_{3}$ substrate) as a function of film thickness measured using a superconducting quantum interference device magnetometer (Quantum Design MPMS). Due to the possible formation of a ferromagnetic europium silicate layer (e.g., $\left.\mathrm{Eu}_{2} \mathrm{SiO}_{4}\right)^{14}$ during silicon deposition on top of $\mathrm{EuTiO}_{3}$ films, uncapped films were measured in Fig. 3. For 200, 100, and $50 \mathrm{~nm}$ thick films, an antiferromagnetic transition occurs at $5.5 \mathrm{~K}$, which corresponds to the antiferromagnetic transition
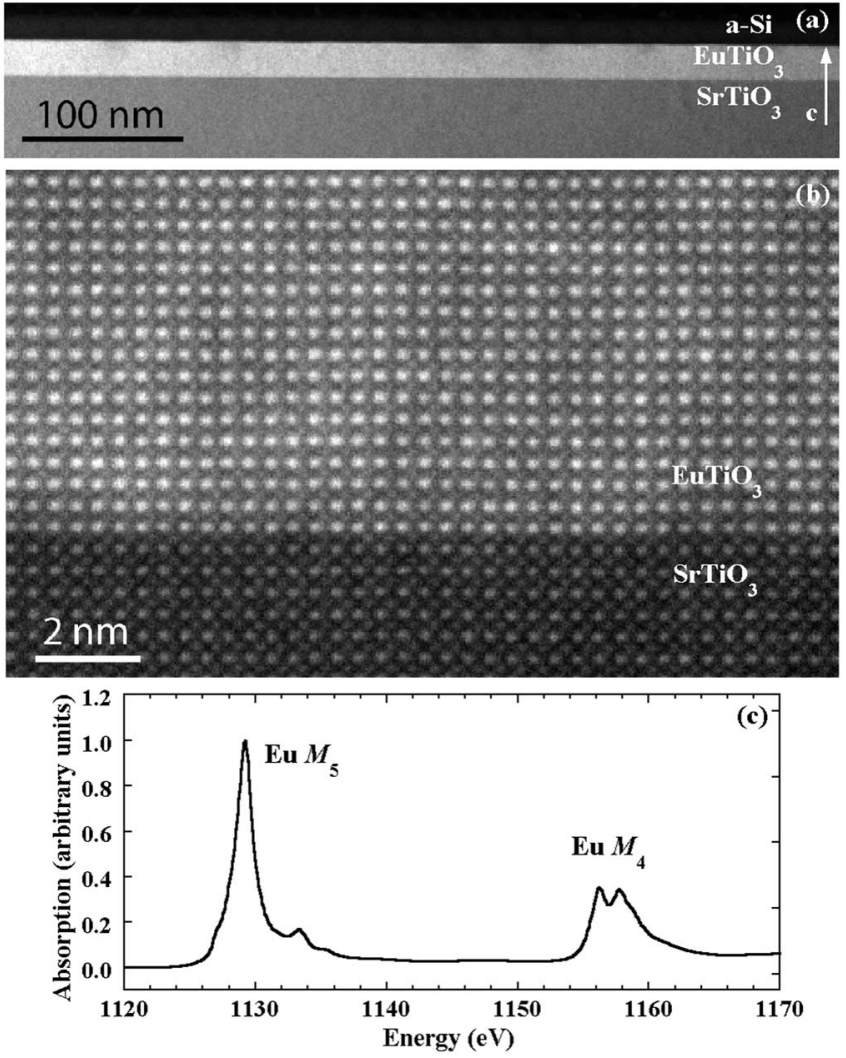

FIG. 2. (a) HAADF STEM image of the same film analyzed in Fig. 1(a). (b) Atomically sharp interface of the $\mathrm{EuTiO}_{3}$ film and the $\mathrm{SrTiO}_{3}$ substrate. (c) Eu $M_{4,5}$ edges from XAS, showing the valence state of europium is $2+$.

temperature $T_{N}$ of bulk $\mathrm{EuTiO}_{3}{ }^{6,7}$ The low field dependence of magnetization for the same $200 \mathrm{~nm}$ thick $\mathrm{EuTiO}_{3}$ film at $1.8 \mathrm{~K}$ is shown in the inset of Fig. 3. The spin-flop transition is indicated by the change of curvature at around $1.5 \mathrm{kOe}$ and no remnant magnetization is observed. The saturation magnetizations (not shown) measured for all films analyzed in Fig. 3 at $1.8 \mathrm{~K}$ under a high magnetic field $(>10 \mathrm{kOe})$ are $6.7 \pm 0.5 \mathrm{Bohr}$ magnetons $\left(\mu_{B}\right)$ per europium atom, which is close to the magnetic moment of a $\mathrm{Eu}^{2+}$ ion $\left(7 \mu_{B} / \mathrm{Eu}\right)$. The bulk-like antiferromagnetic behavior of MBE-grown unstrained $\mathrm{EuTiO}_{3}$ films in combination with RBS and XAS analyses confirms that the MBE-grown films are stoichiometric and have the desired europium valance state. These results support the notion that our growth conditions allow us to investigate the intrinsic magnetic proper-

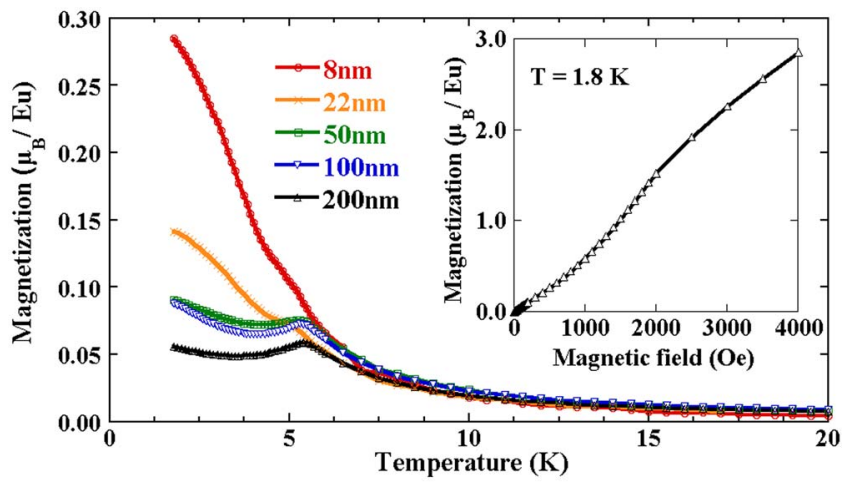

FIG. 3. (Color online) Field-cooled magnetization of 8, 22, 50, 100, and 200 nm thick, uncapped $\mathrm{EuTiO}_{3}$ films at $H=100$ Oe, showing an antiferromagnetic ordering with $T_{N}=5.5 \mathrm{~K}$. (inset) Field dependence of the magnetization for the same $200 \mathrm{~nm}$ thick $\mathrm{EuTiO}_{3}$ film at $1.8 \mathrm{~K}$. 


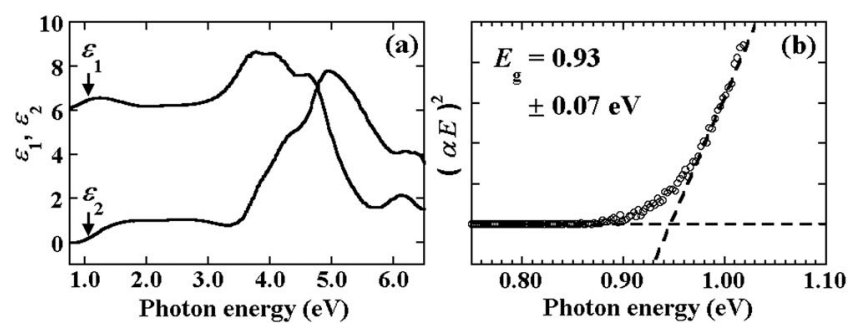

FIG. 4. (a) Dielectric function spectra of an uncapped, $\sim 53 \mathrm{~nm}$ thick $\mathrm{EuTiO}_{3}$ film on (001) $\mathrm{SrTiO}_{3}$ over a spectral range from 0.75 to $6.5 \mathrm{eV}$. (b) Linear extrapolation of $(\alpha E)^{2}=0$ to determine the direct band gap.

ties of $\mathrm{EuTiO}_{3}$ films and that prior results ${ }^{11,12}$ are dominated by extrinsic effects.

The optical band gap of an uncapped, $\sim 53 \mathrm{~nm}$ thick $\mathrm{EuTiO}_{3}$ film grown on (001) $\mathrm{SrTiO}_{3}$ was determined by spectroscopic ellipsometry. Room temperature ellipsometric spectra (in $\Delta$ and $\Psi$ ) were collected ex situ at three angles of incidence, $\Theta_{i}=55^{\circ}, 70^{\circ}$, and $85^{\circ}$, using a variableangle rotating-compensator multichannel spectroscopic ellipsometer ${ }^{24,25}$ over a spectral range from 0.75 to $6.5 \mathrm{eV}$. The complex dielectric function spectra $\left(\varepsilon=\varepsilon_{1}+i \varepsilon_{2}\right)$ shown in Fig. 4(a) were extracted using a least-squares regression analysis and an unweighted root mean square error function $^{26}$ to fit the experimental spectra to a four-medium optical model consisting of a semi-infinite $\mathrm{SrTiO}_{3}$ substrate/ bulk film/surface roughness/air ambient structure. The free parameters correspond to the bulk thickness of the $\mathrm{EuTiO}_{3}$ film, surface roughness, and a parametrization of the $\mathrm{EuTiO}_{3}$ dielectric function, consisting of five Gaussian ${ }^{27}$ oscillators representing the critical point features at energies greater than $3.0 \mathrm{eV}$, two Tauc-Lorentz oscillators ${ }^{28}$ sharing a common Tauc gap below $3.0 \mathrm{eV}$, a high energy Sellemeier oscillator, ${ }^{29}$ and a constant additive term to $\varepsilon_{1}$ represented by $\varepsilon_{\infty}$. The optical properties of the surface roughness layer are represented by a Bruggeman effective medium approximation $^{30}$ consisting of a $50 \%$ bulk film $/ 50 \%$ void mixture. After obtaining a bulk layer thickness of $54.2 \pm 1.3 \mathrm{~nm}$ and a surface roughness thickness of $2.3 \pm 0.4 \mathrm{~nm}$ from the parametrized model, the complex dielectric function spectra were directly obtained by numerical inversion of the $\Theta_{i}=70^{\circ}$ spectra.

The absorption coefficient $\alpha$ is obtained from $\varepsilon$. The onset of optical absorption is observed at $0.85 \pm 0.02 \mathrm{eV}$ for the $\mathrm{EuTiO}_{3}$ film. The direct or indirect band gap may be determined from a linear extrapolation of $(\alpha E)^{2}$ or $(\alpha E)^{1 / 2}$, respectively. ${ }^{31}$ An additional criteria for identification of the indirect band gap involves the presence of two distinct slopes in $(\alpha E)^{1 / 2}$, which is not observed and indicates that $\mathrm{EuTiO}_{3}$ is a direct band gap material. The direct band gap, shown in Fig. 4(b), is at $0.93 \pm 0.07 \mathrm{eV}$. These values are considerably higher than the $E_{g}=0.4 \mathrm{eV}$ gap between the filled europium $f$-level and the empty titanium $d$-level predicted by density functional calculations within the GGA $+\mathrm{U}$ approximation using an appropriate $U-J=5 \mathrm{eV}$ on the europium $f$ orbital determined by Fennie and Rabe. ${ }^{8}$

A comparison between the dielectric functions of $\mathrm{EuTiO}_{3}$ and $\mathrm{SrTiO}_{3}$ shows that each material shares common high energy critical point features, but exhibits different behavior below the indirect band gap of $\mathrm{SrTiO}_{3}$ at $3.2 \mathrm{eV}^{32}$ Below $3.2 \mathrm{eV}, \mathrm{EuTiO}_{3}$ continues to exhibit substantial absorption and shows two additional critical point features at
$1.44 \mathrm{eV}$ and $2.80 \mathrm{eV}$, which are not present in $\mathrm{SrTiO}_{3}$. These features mirror comparisons between the absorption spectra of $\mathrm{SrO}^{33}$ and $\mathrm{EuO},{ }^{34}$ and can be attributed to interactions between $f$ - and $d$-level states.

We gratefully acknowledge the financial support from the National Science Foundation through Grant No. DMR0507146 and the MRSEC Program (Grant Nos. DMR0520404 and DMR-0820404). Work at the Advanced Photon Source is supported by the U.S. Department of Energy, Office of Science, Office of Basic Energy Sciences, under Contract No. DE-AC02-06CH11357.

${ }^{1}$ K. A. Müller and H. Burkard, Phys. Rev. B 19, 3593 (1979).

${ }^{2}$ T. Katsufuji and H. Takagi, Phys. Rev. B 64, 054415 (2001).

${ }^{3}$ J. Brous, I. Fankuchen, and E. Banks, Acta Crystallogr. 6, 67 (1953)

${ }^{4}$ Landolt-Börnstein, in Numerical Data and Functional Relationships in Science and Technology, New Series, Group III, edited by K.-H. Hellwege and A. M. Hellwege (Springer, Berlin, 1981), Vol. 169, p. 59.

${ }^{5}$ B. Ravel and E. A. Stern, Physica B 209, 316 (1995).

${ }^{6}$ T. R. McGuire, M. W. Shafer, R. J. Joenk, H. A. Alperin, and S. J. Pickart, J. Appl. Phys. 37, 981 (1966).

${ }^{7}$ C.-L. Chien, S. DeBenedetti, and F. De S. Barros, Phys. Rev. B 10, 3913 (1974).

${ }^{8}$ C. J. Fennie and K. M. Rabe, Phys. Rev. Lett. 97, 267602 (2006).

${ }^{9}$ R. Ranjan, H. S. Nabi, and R. Pentcheva, J. Phys.: Condens. Matter 19, 406217 (2007).

${ }^{10}$ H.-H. Wang, A. Fleet, J. D. Brock, D. Dale, and Y. Suzuki, J. Appl. Phys. 96, 5324 (2004).

${ }^{11}$ K. Kugimiya, K. Fujita, K. Tanaka, and K. Hirao, J. Magn. Magn. Mater. 310, 2268 (2007).

${ }^{12}$ S. C. Chae, Y. J. Chang, D.-W. Kim, B. W. Lee, I. Choi, and C. U. Jung, J. Electroceram. 22, 216 (2009).

${ }^{13}$ K. Fujita, N. Wakasugi, S. Murai, Y. Zong, and K. Tanaka, Appl. Phys. Lett. 94, 062512 (2009).

${ }^{14}$ M. W. Shafer, J. Appl. Phys. 36, 1145 (1965).

${ }^{15}$ G. J. McCarthy, W. B. White, and R. Roy, J. Inorg. Nucl. Chem. 31, 329 (1969).

${ }^{16}$ Y. S. Kim, D. J. Kim, T. H. Kim, T. W. Noh, J. S. Choi, B. H. Park, and J.-G. Yoon, Appl. Phys. Lett. 91, 042908 (2007).

${ }^{17}$ E. J. Tarsa, E. A. Hachfeld, F. T. Quinlan, J. S. Speck, and M. Eddy, Appl. Phys. Lett. 68, 490 (1996).

${ }^{18}$ T. Ohnishi, K. Shibuya, T. Yamamoto, and M. Lippmaa, J. Appl. Phys. 103, 103703 (2008).

${ }^{19}$ D. G. Schlom, L.-Q. Chen, X. Pan, A. Schmehl, and M. A. Zurbuchen, J. Am. Ceram. Soc. 91, 2429 (2008).

${ }^{20}$ G. Koster, B. L. Kropman, G. Rijnders, D. H. A. Blank, and H. Rogalla, Appl. Phys. Lett. 73, 2920 (1998).

${ }^{21}$ C. D. Theis and D. G. Schlom, J. Vac. Sci. Technol. A 14, 2677 (1996).

${ }^{22}$ L. R. Doolittle, Nucl. Instrum. Methods Phys. Res. B 9, 344 (1985).

${ }^{23}$ T. Kinoshita, H. P. N. J. Gunasekara, T. Takata, S. Kimura, M. Okuno, Y. Haruyama, N. Kosugi, K. G. Nath, H. Wada, M. Shiga, T. Okuda, A. Harasawa, H. Ogasawara, and A. Kotani, J. Phys. Soc. Jpn. 71, 148 (2002).

${ }^{24}$ J. Lee, P. I. Rovira, I. An, and R. W. Collins, Rev. Sci. Instrum. 69, 1800 (1998).

${ }^{25}$ B. D. Johs, J. A. Woollam, C. M. Herzinger, J. N. Hilfiker, R. A. Synowicki, and C. L. Bungay, Proc. SPIE CR 72, 29 (1999).

${ }^{26}$ Y. Cong, I. An, K. Vedam, and R. W. Collins, Appl. Opt. 30, 2692 (1991).

${ }^{27}$ A. D. Rakic and M. L. Majewski, J. Appl. Phys. 80, 5909 (1996).

${ }^{28}$ G. E. Jellison and F. A. Modine, Appl. Phys. Lett. 69, 371 (1996).

${ }^{29}$ R. W. Collins and A. S. Ferlauto, in Handbook of Ellipsometry, edited by H. G. Tompkins and E. A. Irene (William Andrew, Norwich, New York, 2005), pp. 125-129.

${ }^{30}$ H. Fujiwara, J. Koh, P. I. Rovira, and R. W. Collins, Phys. Rev. B 61, 10832 (2000).

${ }^{31}$ J. I. Pankov, Optical Processes in Semiconductors (Dover, New York, 1975), p. 37.

${ }^{32}$ S. Zollner, A. A. Demkov, R. Liu, P. L. Fejes, R. B. Gregory, P. Alluri, J. A. Curless, Z. Yu, J. Ramdani, R. Droopad, T. E. Tiwald, J. N. Hilfiker, and J. A. Woollam, J. Vac. Sci. Technol. B 18, 2242 (2000).

${ }^{33}$ R. L. Sproull, Phys. Rev. 78, 630 (1950).

${ }^{34}$ D. B. Ghosh, M. De, and S. K. De, Phys. Rev. B 70, 115211 (2004). 